\title{
Editorial
}

\section{Modeling, Analysis, and Applications of Complex Systems}

\author{
Chuandong Li, ${ }^{1}$ Xiaodi Li, ${ }^{2}$ Shukai Duan, ${ }^{1}$ and Yanzhi Zhang ${ }^{3}$ \\ ${ }^{1}$ College of Electronic and Information Engineering, Southwest University, Chongqing 400715, China \\ ${ }^{2}$ School of Mathematical Sciences, Shandong Normal University, Jinan 250014, China \\ ${ }^{3}$ Department of Mathematics and Statistics, Missouri University of Science and Technology, Rolla, MO 65409-0020, USA
}

Correspondence should be addressed to Chuandong Li; licd@cqu.edu.cn

Received 12 August 2013; Accepted 12 August 2013

Copyright (C) 2013 Chuandong Li et al. This is an open access article distributed under the Creative Commons Attribution License, which permits unrestricted use, distribution, and reproduction in any medium, provided the original work is properly cited.

Recent advances in theory and applications of complex dynamical systems have contributed much to the successful handling of certain problems in biology, physics, economics, engineering, and so forth that until recently were thought too difficult to be analyzed. These complex systems may be characterized by systems with uncertainty, impulse, time delay, stochastic perturbation, hybrid dynamics, distributed dynamics, and chaotic dynamics. The overall aim of this special issue is to bring together the latest or innovative knowledge and advances in mathematics for handling complex systems, which may depend largely on methods from mathematical analysis, artificial intelligence, statistics, and engineering, including nonlinear dynamics, time series analysis, neural networks, and evolutionary computation. The solicited papers in this special issue should provide solutions to modeling, analysis, control, and applications of real-world complex systems. The topics in this special issue included, but are not limited to, modelling, analysis, and applications of complex systems such as impulsive systems, pulse-modulated systems, switching systems, delayed systems, discontinuous control system theory, bifurcation theory and chaotic dynamics.

The response to this special issue on modelling, analysis, and applications of complex systems was beyond our expectation. We received 39 papers in this research field. This special issue includes thirty-one high-quality peer-reviewed articles. These articles contain some new, novel, and innovative techniques and ideas that may stimulate further research in every branch of theory and applications of complex systems.

\section{Acknowledgments}

We would like to express our gratitude to the reviewers, whose professional comments and valuable suggestions guaranteed the high quality of these selected papers. Also, we appreciate the authors for their interesting and novel contributions. This work is jointly supported by the Natural Science Foundation of China with grant numbers 61374078 and 61372139 .

Chuandong $\mathrm{Li}$

Xiaodi Li

Shukai Duan

Yanzhi Zhang 


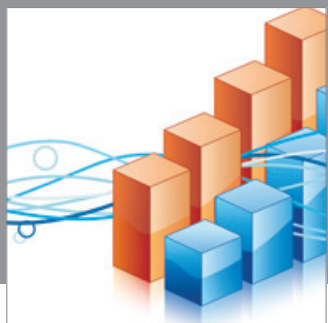

Advances in

Operations Research

mansans

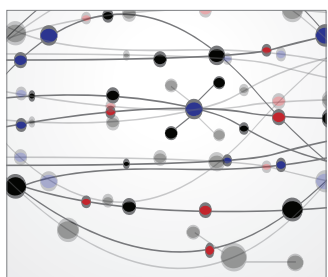

The Scientific World Journal
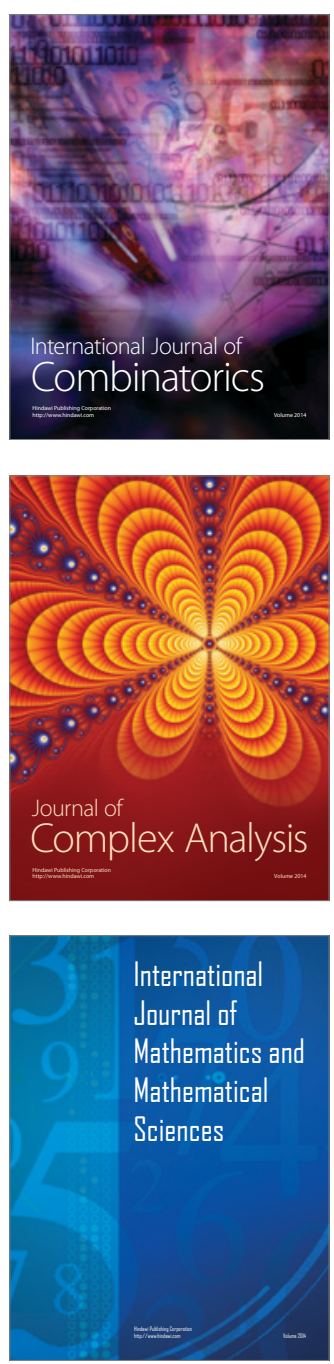
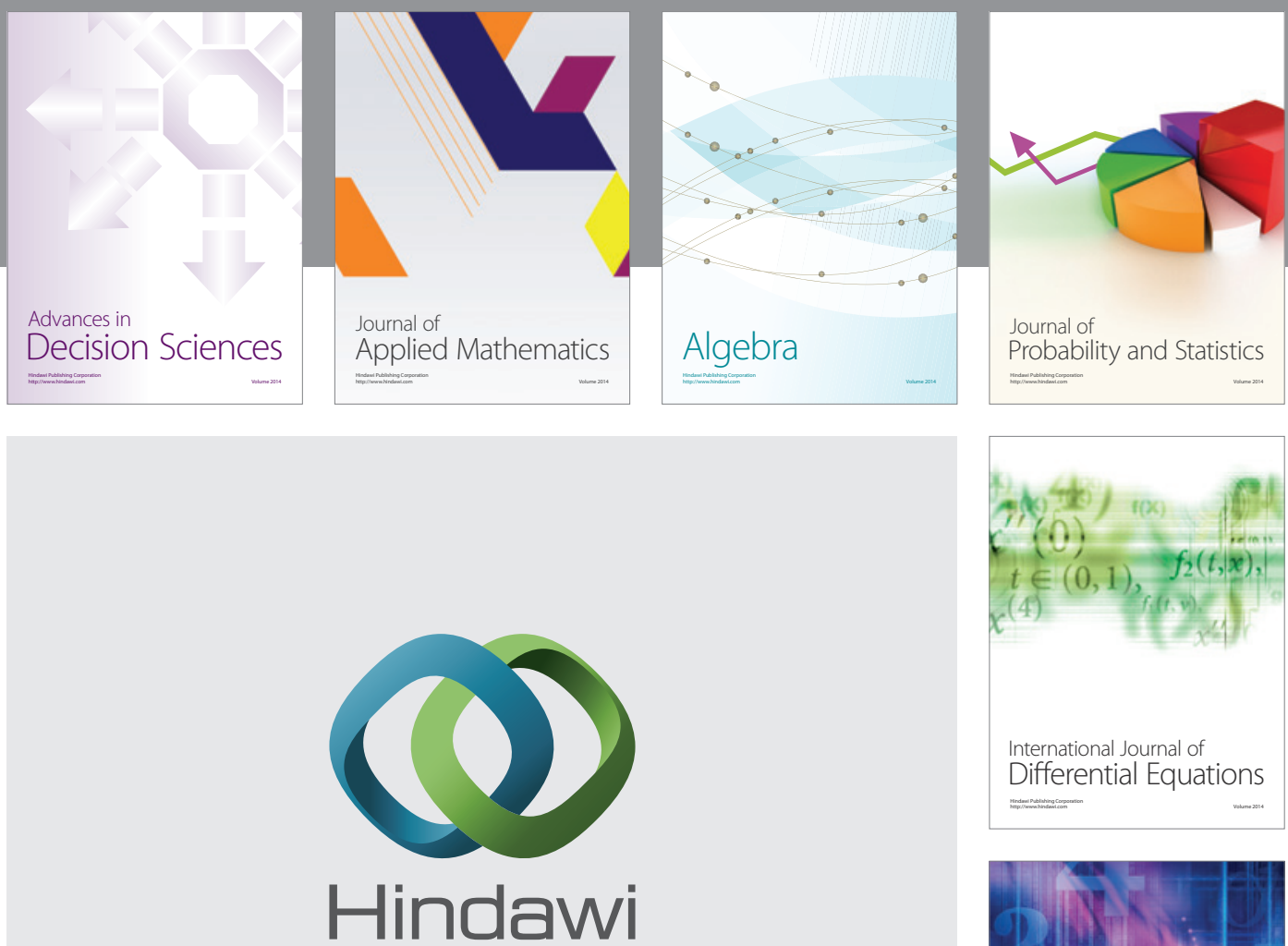

Submit your manuscripts at http://www.hindawi.com
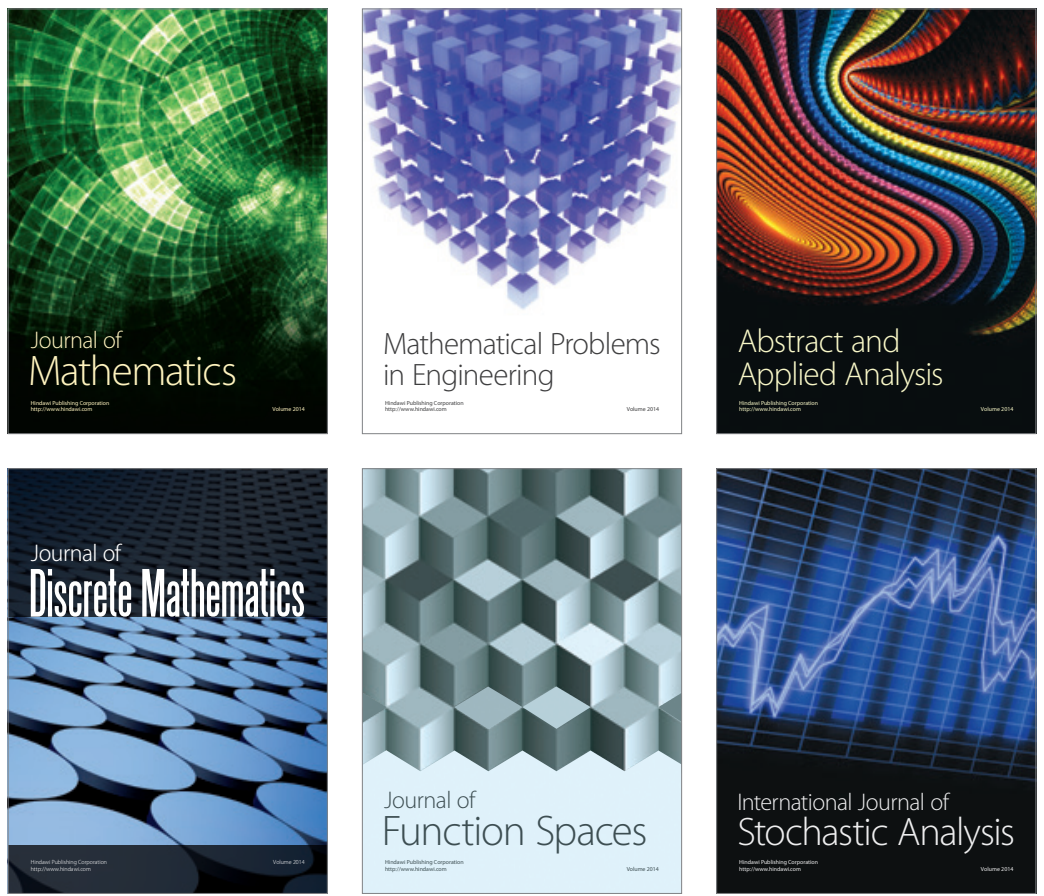

Journal of

Function Spaces

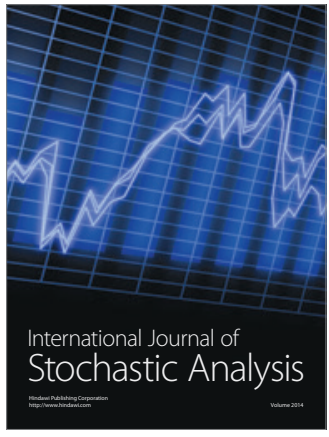

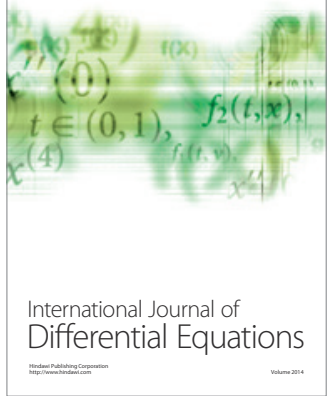
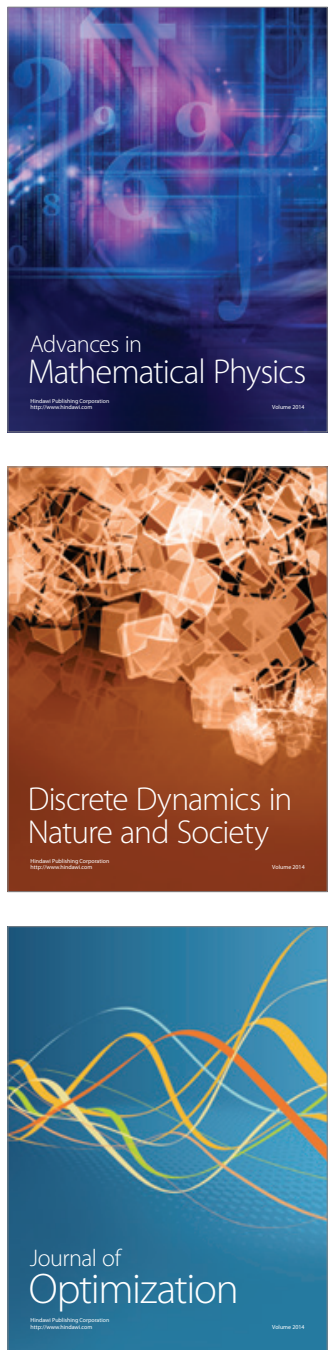